\title{
Determination of Novel Highly Effective Necrostatin Nec-1s in Rat Plasma by High Performance Liquid Chromatography Hyphenated with Quadrupole-Time-of-Flight Mass Spectrometry
}

\author{
Peter Mikuš 1,2,*, Daniel Pecher 1,2 (iD, Drahomíra Rauová 1,2 ${ }^{2}$, Csaba Horváth ${ }^{3}$, Adrián Szobi ${ }^{3}$ \\ and Adriana Adameová 3 \\ 1 Department of Pharmaceutical Analysis and Nuclear Pharmacy, Faculty of Pharmacy, \\ Comenius University in Bratislava, Odbojárov 10, 83232 Bratislava, Slovak Republic; \\ pecher1@uniba.sk (D.P.); rauova@fpharm.uniba.sk (D.R.) \\ 2 Toxicological and Antidoping Center, Faculty of Pharmacy, Comenius University in Bratislava, \\ Odbojárov 10, 83232 Bratislava, Slovak Republic \\ 3 Department of Pharmacology and Toxicology, Faculty of Pharmacy, Comenius University in Bratislava, \\ Odbojárov 10, 83232 Bratislava, Slovak Republic; csaba125@gmail.com (C.H.); \\ adrian.szobi@gmail.com (A.S.); adameova@fpharm.uniba.sk (A.A.) \\ * Correspondence: mikus@fpharm.uniba.sk; Tel.: +421-2-50-117-243
}

Received: 19 July 2018; Accepted: 2 August 2018; Published: 3 August 2018

check for updates

\begin{abstract}
Necrostatins have been shown to retard necroptosis, a programmed necrotic-like cell death, which has been shown to underlie pathophysiology of various diseases. Nec-1s, a novel highly effective necrostatin, overcomes some drawbacks of former necrostatin analogues. The determination of Nec-1s in biological system, however, has not been carried out so far. Therefore, this study was undertaken to optimize and validate the HPLC-DAD-Q-TOF method for the assessment of Nec-1s levels in the plasma what is the necessity for designing its proper dosing regimen for in vivo studies. Benefits of the proposed analytical protocol include: (i) simple sample preparation (precipitation of plasma proteins, evaporation of acetonitrile, reconstitution in mobile phase), (ii) fast, selective and sensitive analysis due to a highly orthogonal LC-MS system providing less than $8 \mathrm{~min}$ analysis time, (iii) detection of Nec-1s without any matrix interferences, and quantitation of very low concentration levels of Nec-1s (LLOQ $\sim 20 \mathrm{ng} / \mathrm{mL}$ ), (iv) high reliability of Nec-1s determination with precision and accuracy values meeting the FDA criteria for biomedical analysis. The proposed analytical protocol is suitable for routine use in relevant biological studies, and, in this work, it was successfully applied for monitoring of Nec-1s plasma levels in rats providing reproducible and consistent results. Based on pharmacokinetic features, which can also be assessed due to the results of this study, there will be efforts to perform both acute and chronic in vivo studies and potential clinical safety studies first.
\end{abstract}

Keywords: high performance liquid chromatography; quadrupole-time-of-flight mass spectrometry; necrostatin Nec-1s; rat plasma; determination; biomedical analysis

\section{Introduction}

Inhibition of receptor interacting protein 1 (RIP1) by a drug class referred to as the necrostatins has been shown to retard necroptosis, a form of programmed necrotic-like cell death which has been suggested to underlie pathophysiology of various diseases, including neurodegenerative diseases [1], acute inflammatory conditions [2], malignancies [3] and cardiovascular diseases associated with ischemia (reviewed by [4,5]). Necrostatin-1 (Nec-1, 5-(indol-3-ylmethyl)(2-thio-3-methyl)hydantoin), 
the first discovered necrostatin [6], which acts as an allosteric inhibitor of kinase domain of RIP1, has been widely used in some of these studies $[4,7,8]$. However, its relatively low kinase selectivity evidenced by the concomitant inhibition of indoleamine 2,3-dioxygenase (IDO), a potent immunomodulatory enzyme [9], may significantly influence the outcomes of such studies, mainly those associated with inflammatory response [10]. In addition, we have indicated that Nec-1, may also influence protein kinases regulating excitation-contraction coupling (ECC) of the heart and thereby its contractile and electrical activity [11]. An inactive analogue, Nec-1i (Nec-1i, N-demethylated thiohydantoin analogue of Nec-1), lacking the capability to inhibit RIP1 and probably also other protein kinases without any effects on ECC [11], can serve as a suitable control. However, this compound was also found to be an IDO inhibitor [10]. In spite of these limitations, other analogues of necrostatin- 1 such as necrostatin-3,-5,-7, which differ in their mechanism of RIP1 inhibition, have not been shown to be superior to Nec-1 and are not routinely used. Instead, 5-((7-chloro-1H-indol-3-yl)methyl)-3-methyl-2,4-imidazolidinedione (Nec-1s), possesses several advantageous pharmacokinetic and pharmacodynamic characteristics in comparison to Nec-1. In fact, it is around two-fold more effective than Nec-1 ( $\mathrm{IC}_{50}=210 \mathrm{nM}$ vs. $494 \mathrm{nM}$ with Nec-1), does not influence IDO and is metabolically more stable (for the chemical structures of Nec-1 and Nec-1s, see Figure 1). Moreover, safety profile of Nec-1s is also superior to that of Nec-1; its in vivo and in vitro toxicity is reduced [10]. Another paper studied use of RIP1 inhibitors in abdominal aortic aneurisms. It was shown that the aortic expansion of mice (with aneurysm induced by elastase perfusion) in case of Nec-1s was significantly smaller than in control $(64.12 \%$ vs. $172.80 \% ; p<0.05)$. In case of Nec-1 the aortic diameter was smaller but the difference was not statistically significant $(p=0.1)$. It was concluded that inhibition of necroptosis with Nec-1s stabilizes pre-existing aneurysms by promoting connective tissue repair and diminishing inflammation [12]. Thus, of all necrostatins, Nec-1s can be considered to be the most preferred RIP1 inhibitor. Unfortunately, not enough information is available about its fate in the organism (e.g., pharmacokinetic characteristics). Therefore, the development of a suitable analytical method for the determination of Nec-1s in biological system is of a high importance.

According to our best knowledge, however, there has been only one method established very recently for the quantitative analysis of necrostatin-1 in rat plasma [13]. The analytical procedure was based on an extraction of the analyte from the sample matrix followed by a HPLC-MS/MS analysis. The extraction recoveries of Nec-1 ranged from $85.40 \%$ to $98.25 \%$, and lower limit of quantification was $10 \mu \mathrm{g} / \mathrm{L}$. The pharmacokinetic study revealed that concentration level of Nec-1 at $5 \mathrm{~min}$ after i.v. administration of the drug was $1733 \mathrm{ng} / \mathrm{mL}$. It was $617 \mathrm{ng} / \mathrm{mL}$ at $1 \mathrm{~h}$, and $<100 \mathrm{ng} / \mathrm{mL}$ at $8 \mathrm{~h}$ after administration. In case of oral administration the peak concentration $(648 \mathrm{ng} / \mathrm{mL})$ was reached at $1 \mathrm{~h}$ after administration and was undetectable after $8 \mathrm{~h}$. The determination of innovative Nec-1s in biological system, however, has not been carried out so far. Therefore, this study was undertaken to optimize and validate the HPLC-DAD-Q-TOF method for the assessment of Nec-1s levels in rat plasma what is needed for designing a proper dosing regimen for in vivo studies.

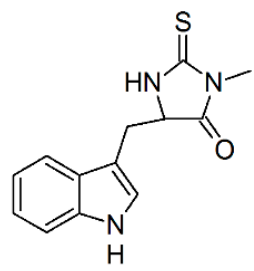

Necrostatin-1

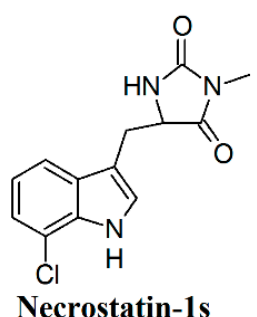

Necrostatin-1s

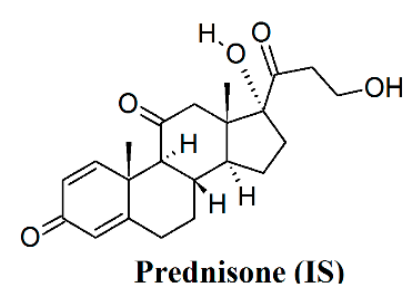

Prednisone (IS)

Figure 1. Chemical structures of necrostatin-1, necrostatin-1s, and prednisone (internal standard, IS). 


\section{Results and Discussion}

The HPLC-DAD-Q-TOF method was systematically optimized (Section 2.1), validated (Section 2.2) and applied for the determination of Nec-1s levels in rat plasma (Section 2.3). The potential of the method for biomedical use is discussed and the results are documented by appropriate figures and tables.

\subsection{Method Development}

The method development included the optimization of both the sample preparation and analytical steps. In the simple sample preparation procedure, described in Section 3.3, the plasma matrix was effectively deproteinized and preconcentrated so that any risk of interferences was reduced and sensitivity of determination enhanced. The HPLC-DAD-Q-TOF method was optimized with respect to the chromatographic separation of Nec-1s from the residual matrix constituents, and, subsequently, UV and MS detection of the pre-separated Nec-1s. A UV and MS detection tandem can be a useful tool in profiling biological matrices as the resulting profiles provide complementary information to the analyzed sample. Therefore, both detection techniques were applied and evaluated for their potentialities in $\mathrm{Nec}-1$ s determination in plasma.

A RP-C18 analytical chromatography column (Zorbax Extend-C18) with dimensions favorable for combination with mass spectrometry $(2.1 \times 50 \mathrm{~mm}, 1.8 \mu \mathrm{m})$ was used in the development of the HPLC-DAD-Q-TOF method. The chromatographic stationary phase was selected with respect to a maximum resolution of the relatively hydrophobic analyte from the residual plasma matrix constituents. A MS-friendly mobile phase, consisting of a mixture of $0.1 \%$ formic acid in water and $0.1 \%$ formic acid in acetonitrile, provided the best mass spectrometer response for Nec-1s. In the method optimization, methanol was also tested as an organic solvent, however, acetonitrile provided a better MS detector response. Similar observations were also made with respect to mobile phase additives. Here, $0.1 \%$ formic acid was optimum compared with $0.1 \%$ acetic acid, ammonium formate with $\mathrm{pH}$ 3.0 (5 mM and $10 \mathrm{mM}$ ), and ammonium acetate with pH 4.0 (5 mM and $10 \mathrm{mM}$ ). Prednisone (for the chemical structure, see Figure 1) was chosen as an internal standard (IS) based on the similarity of its chromatographic properties $(\log P)$ to those of Nec-1s.

A subsequent optimization of the HPLC-DAD-Q-TOF method was devoted to the creation of a proper gradient enabling the highest resolution of Nec-1s from the residual plasma matrix constituents along with the lowest retention time of the analyte. For this purpose different starting compositions of mobile phase $(10-40 \%$ B) and different gradients were evaluated. Based on the peak shapes, retention times of Nec-1s and IS, peak areas and heights, and analyte vs. matrix resolution, the initial composition of mobile phase set at 25\% B and linear gradient to $90 \%$ B in 4 minutes were chosen as optimum. The total analysis time, including column cleaning ( $90 \%$ B for $1 \mathrm{~min})$ and column reconditioning (2.5 min with initial mobile phase composition), was ca. $8.0 \mathrm{~min}$.

The optimized chromatographic conditions resulted in short retention times of Nec-1s and IS ( $<3 \mathrm{~min}$ ), and acceptable peak shapes (see Figure 2). The hyphenated LC-MS method also provided other key benefits such as high sensitivity of Nec-1s determination (see Section 2.2), and no significant/detectable interferences at the elution position of $\mathrm{Nec}-1$ s originating from the plasma matrix (see traces 3a in Figure 2). Detection of the analyte with very high resolution of its mass due to Q-TOF (see Section 3.5) was a prerequisite for avoiding any matrix interference and producing EIC spectra of the analyte with no overlaps (see e.g., panel 3a in Figure 2). On the other hand, in case of the DAD detection (evaluated at a Nec-1s absorption maximum) an interfering signal originating from the residual matrix significantly increased the Nec-1s peak area (see traces $3 b$ in Figure 2). This interference could not be removed even if using different sample preparation procedure (e.g., extraction of Nec-1s with 3:7 hexane-ethyl acetate, unpublished results). Although, due to this interference, the LC-DAD approach was not suitable for the quantitation of Nec-1s at the concentration levels required in the present study, DAD still provided useful information about UV-absorbing compounds appearing in the recorded profiles. In our case, DAD can control absorbing peaks in the elution position of Nec-1s that are not detectable by MS (compare TIC and DAD profiles in panels 3a and 3b of Figure 2). 

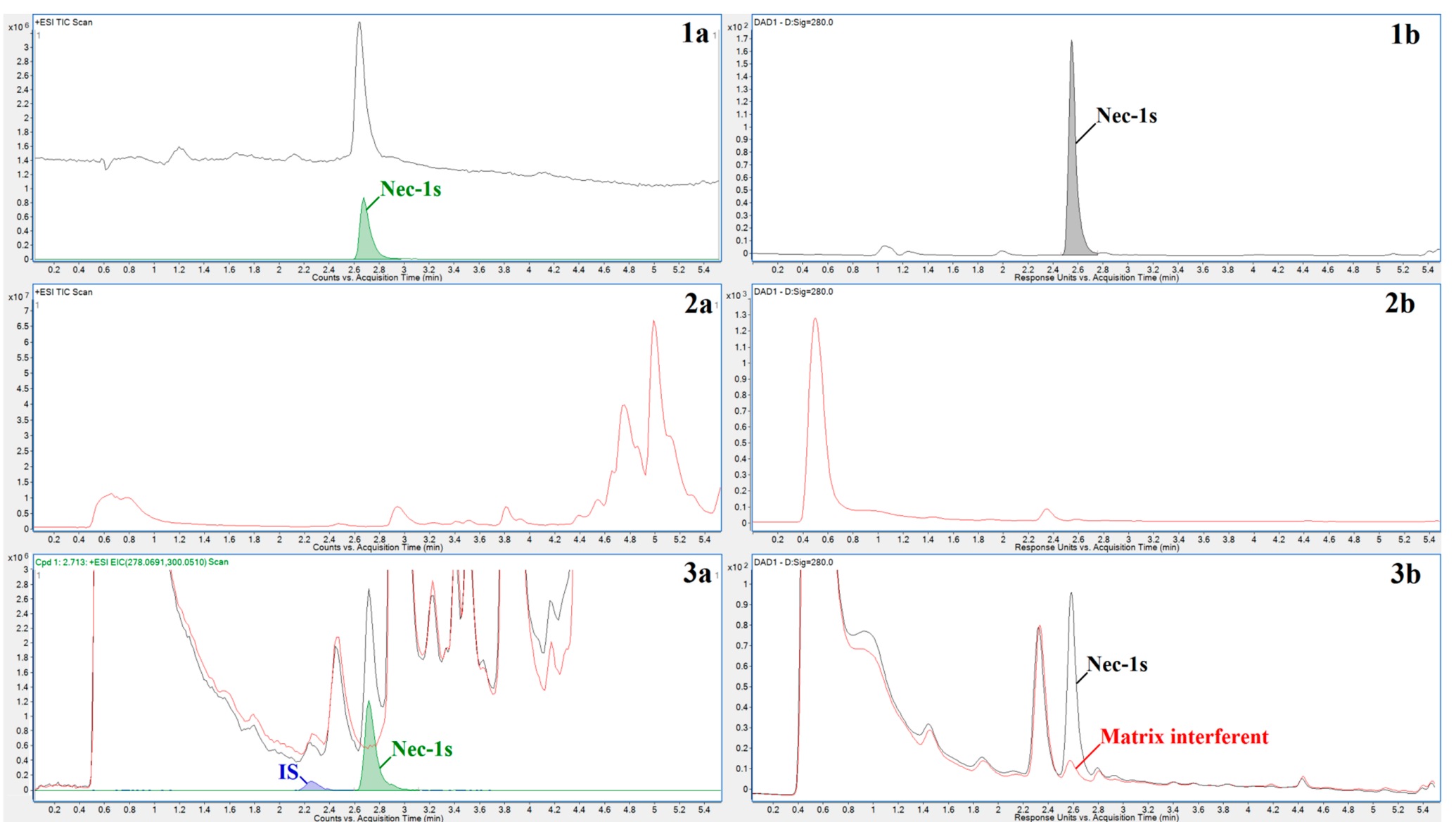

Figure 2. Representative chromatograms of Nec-1s in standard and plasma matrices obtained by the HPLC-DAD-Q-TOF method. MS (a) and DAD (b) profiles of: Nec-1s standard stock solution (1), blank plasma solution-whole chromatogram (2), detail of blank plasma solution (red line) overlapped with spiked plasma solution at a $2030.00 \mathrm{ng} / \mathrm{mL}$ concentration of Nec-1s (black line) (3). Blue and green integrated peaks in (3a) represent EIC of IS and Nec-1s, respectively. Optimum chromatographic and detection (UV, MS) conditions, as given in Sections 3.6 and 3.6, respectively, were used to obtain the present profiles. 
In the next step of the method development the operating parameters of the mass spectrometer (Q-TOF) were optimized in order to obtain maximum ionization and response of the Nec-1s. The operating parameters that were optimized included: Drying gas temperature (evaluated in the range of $200-360{ }^{\circ} \mathrm{C}$ ), drying gas flow (4-12 L/min), nebulizing gas pressure (10-60 psi), capillary voltage (2000-6000 V), fragmentor voltage (50-250 V), and skimmer voltage (20-120 V).

An increase of the drying gas temperature, drying gas flow, and nebulizing gas pressure resulted in an increase of the Nec-1s response (data not shown). Therefore, the maximum possible values (as limited by MS apparatus) were set for these parameters (temp. $360{ }^{\circ} \mathrm{C}$; flow $12 \mathrm{~L} / \mathrm{min}$; pressure $60 \mathrm{psi}$ ). In case of the capillary voltage, fragmentor voltage, and skimmer voltage, the optimum values were chosen based on the highest peak areas obtained during a flow-injection analysis (FIA) of the Nec-1s stock solution (1 $\mu \mathrm{L}$ was injected directly into the MS apparatus). Corresponding dependences are illustrated in Figure 3. Their maxima indicated optimum values for the studied parameters, i.e. $5500 \mathrm{~V}$ for capillary voltage; $125 \mathrm{~V}$ for fragmentor voltage; $65 \mathrm{~V}$ for skimmer voltage.

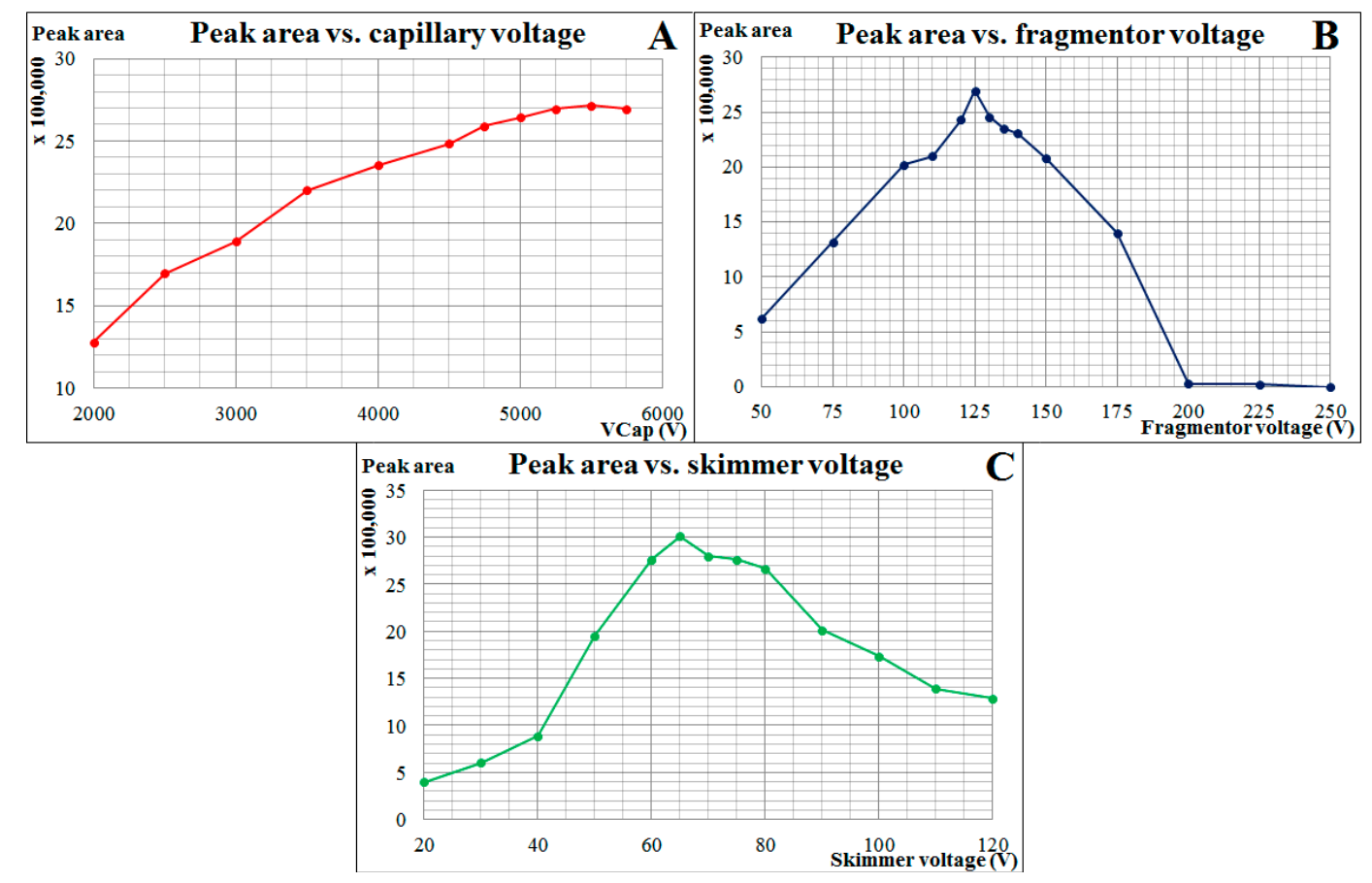

Figure 3. Dependences of Nec-1s detection response on selected MS parameters. FIA experiments were based on direct injection of $1 \mu \mathrm{L}$ of Nec-1s stock solution into the Q-TOF apparatus. Diagrams represent following tested parameters: capillary voltage (A); fragmentor voltage (B); and skimmer voltage (C). Peak areas of Nec-1s were evaluated using its EIC profiles. For other Q-TOF parameters and $m / z$ of the evaluated analyte adducts, see Section 3.5.

\subsection{Method Validation}

The developed HPLC-Q-TOF method with optimized parameters was validated according to the FDA guidelines for bioanalytical methods [14]. Selectivity of the method was evaluated by comparing HPLC-Q-TOF profiles of the blank plasma (prepared as an equimolar mixture from 6 rats) and the same plasma spiked with the reference standard of Nec-1s (see diagram 3a in Figure 2). No interfering peak in the elution position of Nec-1s was observed in the profile of blank plasma. This finding confirmed an acceptable selectivity of the optimized method for monitoring Nec-1s in plasma samples.

Calibration parameters, LLOQ, and retention time of the analyte along with corresponding precision of its determination are listed in Table 1. The Nec-1s and IS peak area ratios were used 
for construction of the calibration line, what effectively minimized influence of fluctuations of some instrumental parameters (e.g., injection volume, column pressure, MS detection response) so that the measurements (elution times, peak areas) were characterized by a high precision (i.e., lower RSD values). The method exhibited excellent linearity in the interval of two decadic orders. The LLOQ value obtained using the Q-TOF detector (50-times higher in comparison to DAD) is favorable for the determination of $\mathrm{Nec}-1 \mathrm{~s}$ on the concentration levels expected after the administration of a therapeutic dose of the drug to rats. Moreover, the method sensitivity can be further increased (at least in one order) when replacing Q-TOF with more sensitive QqQ.

Table 1. Calibration and selected performance parameters of developed HPLC-Q-TOF method for Nec-1s in rat plasma.

\begin{tabular}{|c|c|c|c|c|c|c|c|c|}
\hline \multirow{2}{*}{ RT (min) } & \multirow{2}{*}{$\mathrm{RSD}_{\mathrm{RT}}(\%)$} & \multirow{2}{*}{$\begin{array}{l}\text { Linear Range } \\
\text { (ng/mL) }\end{array}$} & \multirow{2}{*}{ Linearity $r^{2}$} & \multirow{2}{*}{$\begin{array}{l}\text { LLOQ } \\
(\mathrm{ng} / \mathrm{mL})\end{array}$} & \multicolumn{4}{|c|}{ Regression Equation } \\
\hline & & & & & b & SD $^{b}$ & a & $\mathrm{SD}^{\mathrm{a}}$ \\
\hline 2.72 & 0.57 & $20.3-2030.0$ & 0.9991 & 20.3 & 4.062 & 0.028 & -0.014 & 0.023 \\
\hline
\end{tabular}

The intra-day and inter-day precisions and accuracies of the developed method obtained during analysis of the quality control (QC) samples on three concentration levels (low, medium, high) are presented in Table 2. Each QC sample was analyzed 5-times on three consecutive days. The obtained results clearly demonstrated excellent precision (expressed as relative standard deviations, RSD) and accuracy (expressed as relative errors, RE) of the HPLC-Q-TOF method, easily accomplishing the FDA criteria recommended for biomedical analysis.

Table 2. Precision and accuracy of Nec-1s in rat plasma (analysis of QC samples).

\begin{tabular}{ccccc}
\hline \multirow{2}{*}{ QC (ng/mL) } & \multicolumn{2}{c}{ Intra-Day, $\mathbf{n}=\mathbf{5}$} & \multicolumn{2}{c}{ Inter-Day, $\mathbf{n}=\mathbf{1 5}$} \\
\cline { 2 - 5 } & Precision (RSD \%) & Accuracy (RE \%) & Precision (RSD \%) & Accuracy (RE \%) \\
\hline 20.30 & 1.78 & 106.76 & 4.59 & 107.89 \\
203.00 & 1.28 & 108.52 & 1.97 & 106.95 \\
2030.00 & 0.97 & 99.07 & 1.83 & 97.45 \\
\hline
\end{tabular}

The calibration curve was obtained from the analysis of calibration solutions spiked in rat plasma (matrix) and, therefore, the matrix effects and extraction recoveries were accounted for. Despite that, for the illustration, the extraction recoveries and matrix effects were also evaluated on three concentration levels-20.30 ng/mL (low); $203.00 \mathrm{ng} / \mathrm{mL}$ (medium); $2030.00 \mathrm{ng} / \mathrm{mL}$ (high). For the preparation of solutions used for this purpose please refer to Section 3.4. The results from the evaluation of extraction recoveries and matrix effects are presented in Table 3 . The data, ranging in the interval of $89.82-110.42 \%$ (SD $\leq 1.43$ ) for the extraction recoveries and $90.75-109.94 \%$ (SD $\leq 3.60)$ for the matrix effects, are fully acceptable for bioanalytical method.

Table 3. Extraction recoveries and matrix effects of Nec-1s in rat plasma (mean $\pm S D, n=5$ ).

\begin{tabular}{ccc}
\hline QC (ng/mL) & Extraction Recoveries (\%) & Matrix Effects (\%) \\
\hline 20.30 & $89.82 \pm 1.43$ & $109.94 \pm 3.60$ \\
203.00 & $102.58 \pm 1.36$ & $90.75 \pm 0.43$ \\
2030.00 & $110.40 \pm 0.65$ & $97.20 \pm 0.40$ \\
\hline
\end{tabular}

It can be summarized that the performance parameters of the developed HPLC-Q-TOF method, including selectivity, linear range, linearity, sensitivity, accuracy, and precision, are favorable for its biomedical application such as determination of Nec-1s levels in rat plasma (see Section 2.3). 


\subsection{Biomedical Application}

Nec-1s has not been studied in clinical trials so far; only in vitro and ex vivo animal studies have been performed with this agent [10,15-17]. Likewise, according to our best knowledge there are no in vivo animal studies investigating the anti-necroptotic effects of Nec-1s.

The developed and validated HPLC-Q-TOF method was applied for the determination of real plasma concentrations of $\mathrm{Nec}-1 \mathrm{~s}$ in 5 male Wistar rats after i.v. administration of a bolus dose of Nec-1s $(0.37 \mathrm{mg} / \mathrm{kg})$. The samples taken from each subject were prepared and analyzed in triplicate. The experimentally found individual rat plasma levels of Nec-1s are summarized in Table 4. The obtained results clearly demonstrated a suitability of the HPLC-Q-TOF method to monitor trace Nec-1s levels (nM) with a high reproducibility (RSD $<2 \%$ ). The measured data were consistent to each other with plasma concentrations in the range of 319.81-466.52 nM, and mean value of $377.26 \mathrm{nM}$. The differences in the found concentrations can be explained by different metabolism in the studied animals.

When considering the concentration levels found in the pharmacokinetic study of Nec-1 (see the Introduction section) [13], the developed HPLC-Q-TOF method seems to be enough sensitive (see Section 2.2 Method validation) and, by that, easily applicable also for similar pharmacological and clinical studies of Nec-1s (even if Nec-1s exhibits 2-times higher inhibition activity than Nec-1). More specifically, the method could be useful for discovery of relationships and correlations between plasma concentrations of Nec-1s and its selected pharmacological effects (e.g., influence of the contractile and electrical activity of the heart as described in [11], or stabilization of pre-existing aneurisms [12]). Such knowledge could lead to further therapy optimization.

Table 4. Plasma concentrations of Nec-1s found in rats $50 \mathrm{~min}$ after i.v. bolus dose $(0.37 \mathrm{mg} / \mathrm{kg})$ administration.

\begin{tabular}{cccc}
\hline Sample & Plasma Concentration $(\mathbf{n g} / \mathbf{m L})$ & Plasma Concentration $(\mathbf{n M})$ & RSD $\%(\mathbf{n}=3)$ \\
\hline N1 & 103.99 & 374.44 & 0.74 \\
N2 & 90.93 & 327.43 & 1.37 \\
N3 & 110.55 & 398.08 & 0.81 \\
N4 & 88.81 & 319.81 & 0.54 \\
N5 & 129.56 & 466.52 & 0.41 \\
\hline
\end{tabular}

\section{Materials and Methods}

\subsection{Chemicals and Solutions}

The reference standard of Nec-1s was obtained from Merck (Darmstadt, Germany). The reference standard of prednisone ( $\leq 98 \%$; used as internal standard, IS) was obtained from Sigma-Aldrich (Steinheim, Germany). Acetonitrile, methanol and water (all LC-MS grade) were obtained from VWR International GmbH (Vienna, Austria). Formic acid (for mass spectrometry, 98\%) was obtained from Fluka Chemie GmbH (Buchs, Switzerland).

A stock solution of Nec-1s reference standard was prepared at the concentration of $14.5 \mu \mathrm{g} / \mathrm{mL}$ by dissolving the calculated amount of the reference standard in $50 \%$ methanol $(v / v)$. The stock solution of prednisone reference standard (IS) was prepared at the concentration of $50 \mu \mathrm{g} / \mathrm{mL}$ in the same manner. As recommended by the FDA guidelines [14], separate stock solutions were prepared for the QC samples on one side, and for the calibration solutions on the other side. Calibration solutions, QC samples, and all other solutions used in this work were prepared by a proper dilution of the stock solutions. Both Nec-1s and IS stock solutions were stored at $-20{ }^{\circ} \mathrm{C}$ until use.

\subsection{Plasma Sample Collection}

A total of 11 male Wistar rats (250-300 g body weight) were housed under standard conditions with a controlled light/dark cycle $(12 / 12 \mathrm{~h})$ and temperature $\left(2{ }^{\circ} \mathrm{C} \pm 2{ }^{\circ} \mathrm{C}\right)$, and fed a standard diet and tap water ad libitum. The anesthetized animals (natrium pentobarbital $60 \mathrm{mg} / \mathrm{kg}$, i.p.) 
were randomized in two groups to receive either vehicle $(C, n=6)$ or Nec-1s $(N, n=5)$ and blood was collected from the abdominal aorta 50-min after drug application (a bolus dose; $0.37 \mathrm{mg} / \mathrm{kg}$, i.v.; diluted in 20\% v/v DMSO) into EDTA-containing syringes (S-Monovette, Starstedt, Germany). To obtain plasma, the blood was centrifuged at $4{ }^{\circ} \mathrm{C}$ for $15 \mathrm{~min}$ at $4000 \times \mathrm{g}$. The aliquots of plasma were stored at $-80^{\circ} \mathrm{C}$ until further procedure. The dosage of $\mathrm{Nec}-1 \mathrm{~s}$, with respect to its $\mathrm{IC}_{50}(210 \mathrm{nM})$, was chosen to get an equipotent dose to the one used in our previous in vivo experiment [11].

\subsection{Sample Preparation}

An aliquot of plasma was thawed at laboratory temperature. Afterwards, the rat plasma $(180 \mu \mathrm{L})$ was mixed with the internal standard solution $(20 \mu \mathrm{L}, 1.25 \mu \mathrm{g} / \mathrm{mL}$ of prednisone in $50 \%(v / v)$ methanol). The mixture was briefly vortexed and $800 \mu \mathrm{L}$ of acetonitrile was added to the mixture, which resulted in protein precipitation. The precipitated sample was vortexed for $5 \mathrm{~min}(2400 \mathrm{rpm})$ and then centrifuged for $10 \mathrm{~min}\left(15000 \times g ; 4^{\circ} \mathrm{C}\right)$. After the centrifugation, $800 \mu \mathrm{L}$ of supernatant was transferred into another tube and evaporated to dryness under a gentle stream of nitrogen at ambient temperature. The residuals were reconstituted with $100 \mu \mathrm{L}$ of mobile phase (25:75 mixture of acetonitrile and water containing $0.1 \%$ formic acid) and an aliquot of the solution ( $5 \mu \mathrm{L})$ was used for the HPLC-DAD-Q-TOF analysis.

\subsection{Method Validation}

The method's selectivity, linear range, linearity, lower limit of quantification, accuracy, precision, matrix effect, and extraction recovery were evaluated according to the guidelines for bioanalytical methods developed by the US Food and Drug Administration (FDA) authority [14].

The calibration solutions used during the validation of the method were prepared at 6 different concentration levels of Nec-1s $(20.30 ; 50.75 ; 101.50 ; 203.00 ; 507.50$ and $2030.00 \mathrm{ng} / \mathrm{mL})$ by spiking the equimolar mixture of blank plasmas obtained from six rats (receiving only vehicle $\mathrm{C}$ ). The preparation procedure of the calibration solutions (spiked plasma) was the same as for the sample (see Section 3.3). For this purpose, the properly diluted solutions of $\mathrm{Nec}-1 \mathrm{~s}$ reference standard were mixed with both the internal standard solution and blank plasma $(180 \mu \mathrm{L}$ of plasma, $10 \mu \mathrm{L}$ of Nec- $1 \mathrm{~s}, 10 \mu \mathrm{L}$ of $2.5 \mu \mathrm{g} / \mathrm{mL}$ IS). Each calibration solution was analyzed in triplicate.

The calibration curve was prepared using the peak area ratios of $\mathrm{Nec}-1 \mathrm{~s}$ to IS (obtained from the analysis of the calibration solutions with known concentrations) and applying the weighted linear regression (MS Excel 2010). The LLOQ is represented by the lowest concentration in the calibration curve for which the response is at least five times the response compared to blank and the accuracy is within $\pm 20 \%$ of nominal value and precision is not greater than $20 \%$ (according to [14]).

QC samples were prepared at three concentration levels (20.30 (low); 203.00 (medium) and 2030.00 (high) $\mathrm{ng} / \mathrm{mL}$ ) and each was measured five times for three consecutive days in order to evaluate intra-day and inter-day accuracy and precision of the method.

Matrix effects were evaluated by comparing the peak areas obtained by the analysis of plasma samples spiked post-extraction with the peak areas obtained by the analysis of reference standard solution diluted with mobile phase. Extraction recovery was evaluated by comparing the peak areas obtained by the analysis of plasma samples spiked pre-extraction and plasma samples spiked post-extraction. Both the matrix effects and extraction recovery were evaluated at three concentration levels $(20.30 ; 203.00$, and $2030.00 \mathrm{ng} / \mathrm{mL})$.

\subsection{LC-DAD/MS Apparatus}

All of the analyses were carried out on the chromatographic apparatus consisting of LC Agilent Infinity System (Agilent Technologies, Santa Clara, CA, USA) equipped with a gradient pump (1290 Bin Pump VL), an automatic injector (1260 HiPals), and column thermostat (1290 TCC; set at $\left.4{ }^{\circ} \mathrm{C}\right)$. The LC system was hyphenated with a photodiode array detector (Infinity 1290 DAD), and quadrupole time-of-flight (Q-TOF) mass spectrometer (Agilent 6520 Accurate-Mass Q-TOF LC/MS) equipped 
with the electrospray ionization (ESI) source operated in a positive ionization mode. A computer with a MassHunter software (version MassHunter Workstation B.05.01, Agilent Technologies) was used to control the apparatus as well as acquire and process the data. The Q-TOF mass spectrometer was operated under following parameters: drying gas temperature $360^{\circ} \mathrm{C}$, drying gas flow $12 \mathrm{~L} / \mathrm{min}$, nebulizing gas pressure $60 \mathrm{psi}$, capillary voltage $5500 \mathrm{~V}$, fragmentor voltage $125 \mathrm{~V}$, skimmer voltage $65 \mathrm{~V}$, OctopoleRFPeak $550 \mathrm{~V}$, and collision gas $\mathrm{N}_{2}$. The processing of the obtained chromatograms (TIC profiles) resulted in the extracted ion chromatograms (EIC) for Nec-1s and prednisone. In the processing the exact (theoretical) masses of their $[\mathrm{M}+\mathrm{H}]^{+}$(278.0691 for Nec-1s; 359.1853 for prednisone), and $[\mathrm{M}+\mathrm{Na}]^{+}$(300.0510 for Nec-1s; 381.1672 for prednisone) adducts were used. For the MS spectra obtained by the analysis of the stock solutions of Nec-1s and prednisone see Figure 4 . The theoretical and experimentally found masses of the Nec-1s and prednisone adducts were in a very good mutual agreement so that Q-TOF was suitable for an unambiguous confirmation of the identity of the analyzed compounds in the samples. The EIC were extracted with $m / z$ expansion of $\pm 10 \mathrm{ppm}$. In case of DAD detector, the peak areas of the analyte and IS were evaluated at the wavelength of $280 \mathrm{~nm}$.

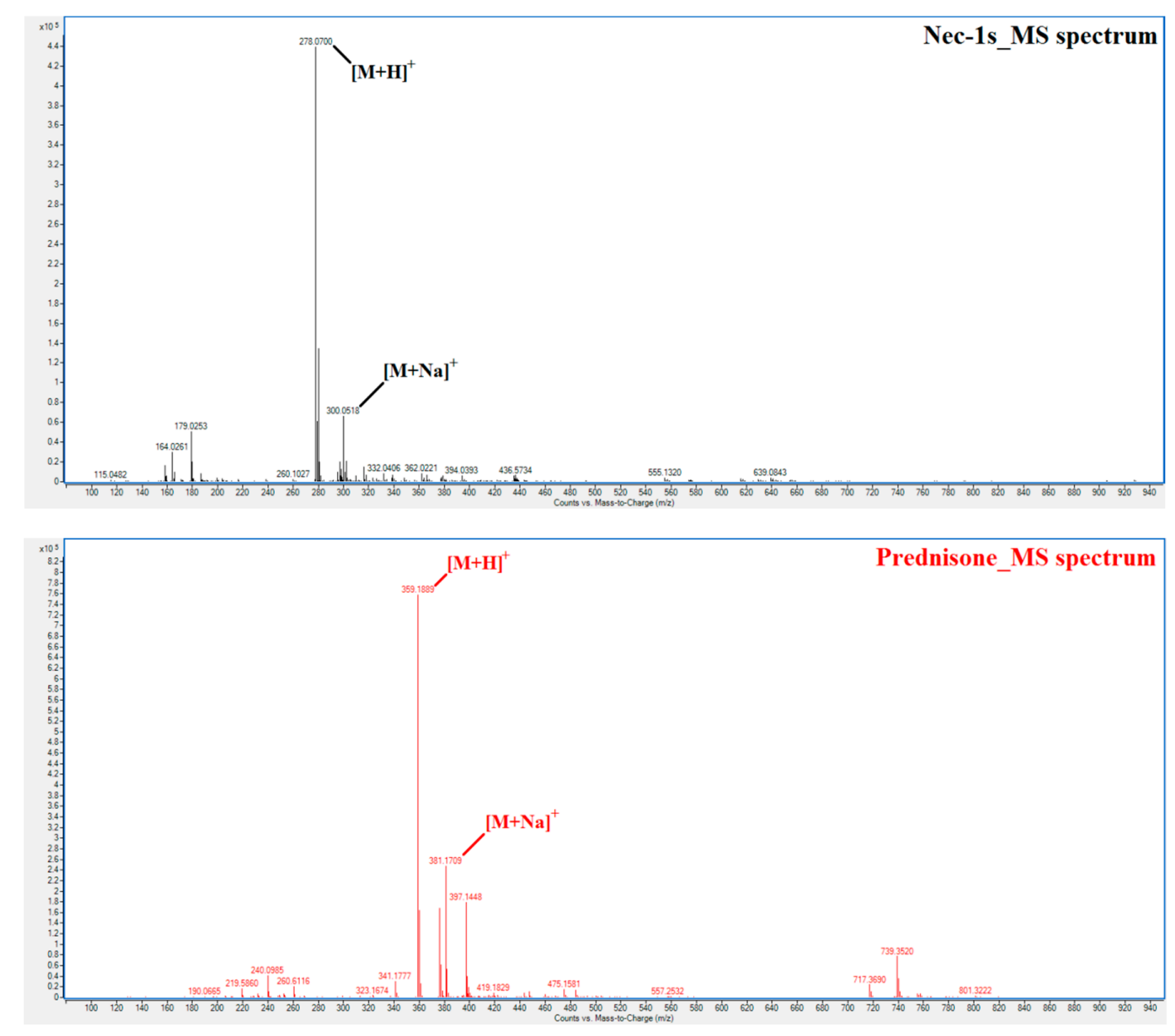

Figure 4. MS spectra of $\mathrm{Nec}-1 \mathrm{~s}$ and prednisone. FIA analysis of $\mathrm{Nec}-1 \mathrm{~s}$ (upper panel) and prednisone (lower panel) stock solutions showing experimental masses for their $\mathrm{H}^{+}$and $\mathrm{Na}^{+}$adducts.

\subsection{Chromatographic Conditions}

The chromatographic separation of the Nec-1s was achieved by using RP-C18 chromatographic column-Zorbax Extend-C18, $2.1 \times 50 \mathrm{~mm}, 1.8 \mu \mathrm{m}$ obtained from Agilent Technologies. A gradient elution of mobile phases consisting of $0.1 \%$ formic acid in LC-MS water (A) and $0.1 \%$ formic acid in 
acetonitrile (B) was used. The gradient elution was set as follows: starting composition of the mobile phases was $25 \% \mathrm{~B}$, which was held for $0.5 \mathrm{~min}$, then increased to $90 \%$ B within next $4.0 \mathrm{~min}$, and held at $90 \% \mathrm{~B}$ for another $1.0 \mathrm{~min}$. The column was reconditioned for $2.5 \mathrm{~min}$ with starting composition of the mobile phases. The flow rate of mobile phases was at $300 \mu \mathrm{L} / \mathrm{min}$ during the whole analysis. The column temperature was maintained at $40{ }^{\circ} \mathrm{C}$.

\section{Conclusions}

These experimental results clearly demonstrated a suitability of the developed and successfully validated HPLC-DAD-Q-TOF method with a simple sample preparation for a highly reliable determination of trace $\mathrm{Nec}-1 \mathrm{~s}$ concentration levels in rat plasma matrices. The presented biomedical application provided reproducible and consistent data for rat plasma levels of Nec-1s, given intravenously as a bolus dose, enabling us to recognize differences in investigated model biological systems (e.g., metabolic activity). The proposed analytical protocol, meeting the FDA criteria, is favorable for routine use, with application areas including therapeutic drug monitoring (for optimization of therapeutic dose) or pharmacokinetic studies of this innovative highly effective drug necrostatin. Further both analytical and pharmacological studies are required to evaluate Nec-1s with respect to its organ selectivity and efficacy in conditions characterized by necroptotic damage.

Author Contributions: P.M. and A.A. proposed and coordinated all the work. A.A., C.H. and A.S. provided the collection and basic treatment of biological samples. D.P. performed the sample preparation, analytical experiments, and data processing. D.R. participated at the sample preparation and analysis. P.M. and D.P. evaluated results and wrote the manuscript. A.A., C.H. and A.S. participated on writing the manuscript. All authors read and approved the final version of the manuscript.

Funding: This work was supported by the projects APVV-15-0585, APVV 15-0607, VEGA 1/0463/18, and VEGA $1 / 0271 / 16$, and carried out in the Toxicological and Antidoping Center at the Faculty of Pharmacy Comenius University in Bratislava. Publication of this work was supported by the publication fund of the Faculty of Pharmacy Comenius University in Bratislava.

Conflicts of Interest: The authors declare that they have no conflict of interest. The funders had no role in the design of the study; in the collection, analyses, or interpretation of data; in the writing of the manuscript, and in the decision to publish the results.

\section{References}

1. Zhang, S.; Tang, M.; Luo, H.; Shi, C.; Xu, Y. Necroptosis in neurodegenerative diseases: A potential therapeutic target. Cell Death Dis. 2017, 8, e2905. [CrossRef] [PubMed]

2. Pasparakis, M.; Vandenabeele, P. Necroptosis and its role in inflammation. Nature 2015, 517, 311-320. [CrossRef] [PubMed]

3. Wang, T.; Jin, Y.; Yang, W.; Zhang, L.; Jin, X.; Liu, X.; He, Y.; Li, X. Necroptosis in cancer: An angel or a demon? Tumor Biol. 2017, 39, 1-11. [CrossRef] [PubMed]

4. Adameova, A.; Goncalvesova, E.; Szobi, A.; Dhalla, N.S. Necroptotic cell death in failing heart: Relevance and proposed mechanisms. Heart Fail. Rev. 2016, 21, 213-221. [CrossRef] [PubMed]

5. Adameova, A.; Hrdlicka, J.; Szobi, A.; Farkasova, V.; Kopaskova, K.; Murarikova, M.; Neckar, J.; Kolar, F.; Ravingerova, T.; Dhalla, N.S. Evidence of necroptosis in hearts subjected to various forms of ischemic insults. Can. J. Physiol. Pharmacol. 2017, 95, 1163-1169. [CrossRef] [PubMed]

6. Degterev, A.; Huang, Z.; Boyce, M.; Li, Y.; Jagtap, P.; Mizushima, N.; Cuny, G.D.; Mitchison, T.J.; Moskowitz, M.A.; Yuan, J. Chemical inhibitor of nonapoptotic cell death with therapeutic potential for ischemic brain injury. Nat. Chem. Biol. 2005, 1, 112-119. [CrossRef] [PubMed]

7. Linkermann, A.; Hackl, M.J.; Kunzendorf, U.; Walczak, H.; Krautwald, S.; Jevnikar, A.M. Necroptosis in immunity and ischemia-reperfusion injury. Am. J. Transplant. 2013, 13, 2797-2804. [CrossRef] [PubMed]

8. Galluzzi, L.; Kepp, O.; Chan, F.K.-M.; Kroemer, G. Necroptosis: Mechanisms and relevance to disease. Annu. Rev. Pathol. Mech. Dis. 2017, 12, 103-130. [CrossRef] [PubMed]

9. Soliman, H.; Mediavilla-Varela, M.; Antonia, S. Indoleamine 2,3-Dioxygenase: Is it an immune suppressor? Cancer J. 2010, 16, 354-359. [CrossRef] [PubMed] 
10. Takahashi, N.; Duprez, L.; Grootjans, S.; Cauwels, A.; Nerinckx, W.; DuHadaway, J.B.; Goossens, V.; Roelandt, R.; Van Hauwermeiren, F.; Libert, C.; et al. Necrostatin-1 analogues: Critical issues on the specificity, activity and in vivo use in experimental disease models. Cell Death Dis. 2012, 3, e437. [CrossRef] [PubMed]

11. Szobi, A.; Rajtik, T.; Adameova, A. Effects of necrostatin-1, an inhibitor of necroptosis, and its inactive analogue Nec-1i on basal cardiovascular function. Physiol. Res. 2016, 65, 861-865. [PubMed]

12. Wang, Q.; Zhou, T.; Liu, Z.; Ren, J.; Phan, N.; Gupta, K.; Stewart, D.M.; Morgan, S.; Assa, C.; Kent, K.C.; et al. Inhibition of receptor-interacting protein kinase 1 with necrostatin-1s ameliorates disease progression in elastase-induced mouse abdominal aortic aneurysm model. Sci. Rep. 2017, 7, 42159. [CrossRef] [PubMed]

13. Geng, F.; Yin, H.; Li, Z.; Li, Q.; He, C.; Wang, Z.; Yu, J. Quantitative analysis of necrostatin-1, a necroptosis inhibitor by LC-MS/MS and the study of its pharmacokinetics and bioavailability. Biomed. Pharmacother. 2017, 95, 1479-1485. [CrossRef] [PubMed]

14. Bioanalytical Method Validation. In Food and Drug Administration Guidance for Industry; U.S. Department Health Human Services: Washington, DC, USA, 2001.

15. Teng, X.; Degterev, A.; Jagtap, P.; Xing, X.; Choi, S.; Denu, R.; Yuan, J.; Cuny, G.D. Structure-activity relationship study of novel necroptosis inhibitors. Bioorg. Med. Chem. Lett. 2005, 15, 5039-5044. [CrossRef] [PubMed]

16. Degterev, A.; Maki, J.L.; Yuan, J. Activity and specificity of necrostatin-1, small-molecule inhibitor of RIP1 kinase. Cell Death Differ. 2013, 20, 366. [CrossRef] [PubMed]

17. Szobi, A.; Farkašová-Ledvényiová, V.; Lichý, M.; Muráriková, M.; Čarnická, S.; Ravingerová, T.; Adameová, A. Cardioprotection of ischaemic preconditioning is associated with inhibition of translocation of MLKL within the plasma membrane. J. Cell. Mol. Med. 2018, 1-14. [CrossRef] [PubMed]

Sample Availability: Samples of the compounds are not available from the authors.

(C) 2018 by the authors. Licensee MDPI, Basel, Switzerland. This article is an open access article distributed under the terms and conditions of the Creative Commons Attribution (CC BY) license (http://creativecommons.org/licenses/by/4.0/). 